\title{
LARVICIDAL EFFICACIES OF FOUR INDIGENOUS PLANT EXTRACTS AGAINST RED FLOUR BEETLE, TRIBOLIUM CASTANEUM (HERBST) (COLEOPTERA: TENEBRIONIDAE)
}

\author{
MD. ADNAN AL BACHCHU ${ }^{1}$, KISMOT ARA ${ }^{2}$, MD. NIZAM UDDIN AND ROUSHAN ARA ${ }^{3}$ \\ Department of Entomology, Hajee Mohammad Danesh Science and Technology University \\ (HSTU), Dinajpur, Bangladesh
}

\begin{abstract}
An investigation was carried out on the larvicidal efficacy of four indigenous plant extracts against the $6^{\text {th }}$ instar larvae of red flour beetle, Tribolium castaneum (Herbst) during the period of February to July 2016. The result revealed that all the four plant extracts were effective in checking insect infestation and had different toxic effects against the $6^{\text {th }}$ instar larvae of red flour beetle. Average mortality indicated that castor plant extract showed the highest toxic effect (average mortality 57.78\%) whereas the lowest toxicity (average mortality $27.46 \%$ ) was found in the neem leaf extracts. The larval mortality significantly differed among all the concentrations of the plant extracts applied and the highest mortality $(60.75 \%)$ was recorded in maximum concentration $\left(10.61 \mathrm{mg} / \mathrm{cm}^{2}\right)$ of the plant extract. No larval mortality was observed in untreated control up to 72 HATs. Mortality percentage was also observed directly proportional to the level of concentrations of plant extracts and to the exposure period. Probit analysis of larval mortality also revealed that the castor plant extract showed the highest toxicity at different time interval among all the plant extracts applied.
\end{abstract}

Key words: Plant extracts, Larvicide, Mortality, Toxicity, Tribolium castaneum

\section{Introduction}

Stored agricultural products are attacked by more than 600 species of beetles, 70 species of moths and about 355 species of mites which cause quantitative and qualitative losses (Rajendran and Sriranjini 2008). These insect pests are the major cause of grain loss during post harvest storage, particularly in the tropical countries (Rajashekar et al. 2012). It was estimated that more than one-third of the food products are lost due to various pests during post-harvest storage (Tripathi et al. 2009). During storage food grain losses due to insect infestation are the most serious problem, specifically in the developing countries like Bangladesh (Dubey et al. 2008). Among the storage pests, Red flour beetle, Tribolium castaneum (Herbst) (Coleoptera: Tenebrionidae) is one of the most comprehensive and destructive pests of stored products, feeding on different stored grain and grain products such as peas, beans, cocoa, nuts, dried fruits and spices but milled grain products such as flour is the most preferred food (Mishra et al. 2012a and 2012b). They are attracted to grain with high moisture content where they encourage mold growth and produce a displeasing, musty odor. 
Currently, different kinds of preventive and curative control measures are practiced to protect insect pests. Among them, chemical pesticides have been used for a long time, but have serious drawbacks (Sharaby 1988). Indiscriminate and continuous use of chemical insecticides create several problems in agroecosystem such as direct toxicity to beneficial insects, fishes and human (Goodland et al. 1985), pesticide resistance, increased environmental and social costs (Pimentel et al. 1980). To overcome these problems, it is utmost necessary to search the alternative pest control methods. The botanical pesticides which defend stored grains from pests are very helpful because of diverse distinct advantages. Botanical pesticides are of broad spectrum, safe to apply, unique in action and can be easily processed. Maximum botanical insecticides are non-hazardous and nontoxic to human. The main advantages of botanicals are that they are easily produced by farmers and are less expensive.

Interest in the use of botanical insectecides has increased over the past years (Isman 2000). Scientists in different parts of the world are working for the upliftment and induction of plant based pesticide, generally known as phytopesticide, botanical pesticide, biopesticide or natural pesticides (Siddiqui et al. 2009). Plant extracts contain compounds that show ovicidal, repellent, antifeedant and toxic effects in insects (Isman 2006). Previously investigations had been conducted on the toxicity effects of indigenous plant extracts against the adult stages of red flour beetle (Mahdeem 1998, Morgan 2009 and Joel 2015) but few research works had focused on the efficacies of plant extracts directly on the larval mortality of red flour beetle (Khalequzzaman and Sultana, 2006 and Yasir et al. 2012). Therefore, the present study was undertaken to evaluate the larvicidal efficacy of neem, thorn apple, castor and custard apple plant extracts against the larvae of Tribolium castaneum.

\section{Materials and Methods}

The present study on the larvicidal efficacies of four indigenous plant extracts against red flour beetle, Tribolium castaneum (Herbst) was conducted in the laboratory of the Department of Entomology, Hajee Mohammad Danesh Science and Technology University (HSTU), Dinajpur during the period of February to July 2016.

Collection and preparation of plant extract: The fresh mature plant leaves of custard apple (Annona reticulata), neem (Azadirachta indica), castor (Ricinus communis) and thorn apple (Datura stramonium) were collected from the HSTU campus, Dinajpur and the nearest area. Collected plant materials were air dried in shade followed by one day sun dried for 4 hours. The dried leaves were made powder separately by an electric grinder in the laboratory. The dust was passed through a $60 \mu \mathrm{m}$ mesh sieve to get fine powder. The powdered leaves were extracted in the methanol solvent. Hundred gram of every plant powders were taken separately in a $500 \mathrm{ml}$ conical flask and mixed with 300 $\mathrm{ml}$ of methanol. The mixture was stirred for 30 minutes and then allowed to shake in the shaker machine for 24 hours. Next they were filtered through a filter paper (Whatman no. 1) and allowed to evaporate the solvents in the vacuum rotary evaporator and finally 
hard different color extracts were collected. The collected crude extracts were preserved in tightly corked vials and stored in a refrigerator for further experimental use.

Preparation of food medium: Standard mixture of wheat flour with powdered dry yeast in a ratio of 19:1 was used as food medium (Park 1962). Food medium was sterilized at $60{ }^{\circ} \mathrm{C}$ temperature for 6 hours in an oven. After sterilization food was not used until at least 15 days to recover its moisture contents (Mondal 1984). In the micrometer sieve, both flour and yeast were passed through and then were mixed thoroughly for homogeneous mixing.

Collection and rearing of Tribolium castaneum: Adult beetles were collected from naturally infected wheat flour from the local market of Dinajpur town. Beetles were reared in glass beaker $(500 \mathrm{ml})$ with the food medium (wheat flour). The beakers were kept in an incubator at $30 \pm 0.5^{\circ} \mathrm{C}$ temperature without light and humidity control. About 500 adults in each beaker were introduced with $500 \mathrm{~g}$ of wheat flour. The cultures were checked regularly and eggs along with larvae were separated to increase the population properly. A crumpled filter paper was placed inside the beaker for the easy movement of the bettles as well as to avoid the cannibalism of eggs. The beakers were covered with pieces of muslin cloth tightly fixed with the help of rubber bands to avoid possible escape of the beetles.

Determination of larval instars: About 500 beetles from the culture were placed in a beaker containing standard food medium. The beaker was covered with a cloth and kept in an incubator at $30{ }^{\circ} \mathrm{C}$ for egg collection. In regular interval, the eggs were collected by sieving the food medium by two sieves of 500 and $250 \mu \mathrm{m}$ mesh separating the adults and eggs, respectively following the methods of Khan and Selman (1981). For egg collection, mainly $250 \mu \mathrm{m}$ sieves were used. The collected eggs were kept in $60 \mathrm{~mm}$ in diameter petridishes and incubated at the same temperature $\left(30{ }^{\circ} \mathrm{C}\right)$. Eggs hatched after 3 - 5 days and the newly hatched larvae were collected with a fine camel hair brush and then transferred to the fresh food medium for culture. The larval instars were determined by counting the number of exuviate (larval skin) deposited in the food medium (Mondal 1984). The $2^{\text {nd }}$ day larvae were found as first instar larvae while the second, third, fourth, fifth and sixth instar larvae were found from the larval culture on $3^{\text {rd }}, 6^{\text {th }}, 9^{\text {th }}, 12^{\text {th }}$ and $16^{\text {th }}$ day after hatching, respectively. Larval culture was also maintained in an incubator in the temperature at $30^{\circ} \mathrm{C} \pm 0.5^{\circ} \mathrm{C}$ without light and humidity control. The food medium was replaced by three days interval to avoid conditioning by the larvae.

Bioassay test (mortality test): Residual film method (Busvine 1971) was used to larvicidal test of different plant extracts against the $6^{\text {th }}$ instar larvae of Tribolium castaneum (Herbst). One $\mathrm{ml}$ plant extract of each dose was dropped separately on petridishes $(60 \mathrm{~mm})$ with the help of pipette, covering uniformly the whole area of the petridish internally. The petridishes were then kept open for sometimes to evaporate the 
solvents fully. Then 10 larvae of $6^{\text {th }}$ instar were released in each petridish. Only methanol solvent was used for the control treatment. Three replications were made for each dose of all the treatments. The petridishes were then kept without food in the laboratory and larval mortality was recorded at 12, 24, 36, 48, 60 and 72 hours after treatments (HAT). The percentage of mortality was corrected using Abbott's formula (Abbott 1987).

$$
P=\frac{p^{1}-C}{100-C} \times 100
$$

Where,

$$
\begin{aligned}
& P=\text { Percentage of corrected mortality } \\
& P^{\prime}=\text { Observed mortality }(\%) \\
& \mathrm{C}=\text { Mortality }(\%) \text { at control. }
\end{aligned}
$$

Statistical analysis: The experiment was conducted using CRD. The data obtained from the experiments were statistically analyzed by MSTATC computer program. The significance of the mean difference was tested by DMRT. The observed mortality was also subjected to probit analysis.

\section{Results and Discussion}

The results of the experiment are presented and discussed under the following subheadings:

Toxicity effect of different plant extracts against $6^{\text {th }}$ instar larvae of red flour beetle: Average mortality percentages of $6^{\text {th }}$ instar larvae of red flour beetle at $12,2436,48,60$ and 72 hours after treatment (HAT) indicated that castor plant extract showed the highest (average mortality, 57.78\%) toxic effect, whereas neem plant extract showed the lowest (average mortality, 27.46\%) toxic effect (Table 1). Mortality percentages of four plant extracts were directly proportional to the time after treatment. There was significant difference $(\mathrm{p}<0.01)$ among the toxicity effects of plant extracts applied on the $6^{\text {th }}$ instar larva of T. castaneum. The order (highest to lowest) of toxicity effect of four plant extracts against the $6^{\text {th }}$ instar larvae of red flour beetle were: castor > custard apple > thorn apple > neem. Mortality percentage also differed significantly among all the concentration level at different time interval (Table 2). The highest mortality $(60.75 \%)$ was at the maximum concentration $\left(10.61 \mathrm{mg} / \mathrm{cm}^{2}\right)$ of plant extract. Mortality percentage is directly proportional to the level of concentration. The interaction of different plant extracts of different doses at different time interval indicated that castor plant extracts showed the highest average per cent larval mortality at maximum dose $\left(10.61 \mathrm{mg} / \mathrm{cm}^{2}\right)$ which was statistically different from all other plant extract at different concentration level (Table 3). Mortality percentages of red flour beetle of different plant extracts of different dose level at different hours were found statistically significant. 
Table 1 . Toxicity effect of different plant extracts against $6^{\text {th }}$ instar larvae of Tribolium castaneum at different HAT (interaction of plant extracts and time).

\begin{tabular}{llllllll}
\hline \multirow{2}{*}{$\begin{array}{l}\text { Plant extracts } \\
\text { used }\end{array}$} & \multicolumn{7}{c}{ Percentage of larval mortality at different time intervals } \\
\cline { 2 - 8 } & 12HAT & 24HAT & 36HAT & $48 \mathrm{HAT}$ & $60 \mathrm{HAT}$ & $72 \mathrm{HAT}$ & $\begin{array}{l}\text { Average } \\
\text { mortality }\end{array}$ \\
\hline Neem & $6.667 \mathrm{c}$ & $17.22 \mathrm{c}$ & $22.78 \mathrm{c}$ & $31.48 \mathrm{~d}$ & $35.80 \mathrm{c}$ & $50.79 \mathrm{c}$ & $27.46 \mathrm{~d}$ \\
Thorn apple & $9.444 \mathrm{bc}$ & $16.11 \mathrm{c}$ & $28.33 \mathrm{c}$ & $36.79 \mathrm{c}$ & $43.83 \mathrm{~b}$ & $60.26 \mathrm{~b}$ & $32.46 \mathrm{c}$ \\
Custard apple & $12.78 \mathrm{~b}$ & $24.44 \mathrm{~b}$ & $42.22 \mathrm{~b}$ & $51.67 \mathrm{~b}$ & $73.27 \mathrm{a}$ & $79.88 \mathrm{a}$ & $47.38 \mathrm{~b}$ \\
Castor & $22.22 \mathrm{a}$ & $43.89 \mathrm{a}$ & $55.56 \mathrm{a}$ & $70.00 \mathrm{a}$ & $75.80 \mathrm{a}$ & $79.20 \mathrm{a}$ & $57.78 \mathrm{a}$ \\
\hline LSD & 4.104 & 4.606 & 6.368 & 5.182 & 4.340 & 3.996 & 2.652 \\
CV $(\%)$ & 47.92 & 27.04 & 25.53 & 16.28 & 11.32 & 8.83 & 9.59 \\
s $_{-}$ & 1.443 & 1.620 & 2.240 & 1.822 & 1.526 & 1.405 & 0.9327 \\
x & & & & & & & \\
\hline
\end{tabular}

HAT $=$ Hour after treatment. Within column values followed by different letter(s) are significantly different at $5 \%$ level of probability by DMRT.

From the above result, it is apparent that among the tested four plant extracts, castor showed the most toxic effect against the $6^{\text {th }}$ instar larvae of the red flour beetle. The reduction of larval population by using the leaf extracts of castor is similar to the previous findings of Basheer (2014). He reported that the castor leaf extract obtained as the best with the mortality of the larvae of Anopheles arabiensis was $96 \%$ after 24 hours with an $\mathrm{LC}_{50}$ at $0.390 \mathrm{mg} / \mathrm{l}, 100 \%$ mortality was observed after 48 hours with $\mathrm{LC}_{50}$ at $0.284 \mathrm{mg} / \mathrm{l}$. Collavino et al. (2006) reported that castor bean leaf powder is effective

Table 2. Toxicity effect of different doses of plant extracts against $6^{\text {th }}$ instar larvae of Tribolium castaneum at different HAT (interaction of dose and time).

\begin{tabular}{llllllll}
\hline & \multicolumn{7}{c}{ Percentage of larval mortality at different time intervals } \\
\cline { 2 - 7 } & $12 \mathrm{HAT}$ & $24 \mathrm{HAT}$ & $36 \mathrm{HAT}$ & $48 \mathrm{HAT}$ & $60 \mathrm{HAT}$ & $72 \mathrm{HAT}$ & $\begin{array}{c}\text { Average } \\
\text { mortality }\end{array}$ \\
\hline 10.61 & $21.67 \mathrm{a}$ & $40.83 \mathrm{a}$ & $57.50 \mathrm{a}$ & $68.52 \mathrm{a}$ & $80.74 \mathrm{a}$ & $95.23 \mathrm{a}$ & $60.75 \mathrm{a}$ \\
5.30 & $18.33 \mathrm{ab}$ & $35.83 \mathrm{a}$ & $52.50 \mathrm{a}$ & $63.24 \mathrm{ab}$ & $76.95 \mathrm{a}$ & $89.06 \mathrm{~b}$ & $55.99 \mathrm{~b}$ \\
2.65 & $15.00 \mathrm{bc}$ & $29.17 \mathrm{~b}$ & $43.33 \mathrm{~b}$ & $58.98 \mathrm{~b}$ & $67.87 \mathrm{~b}$ & $80.78 \mathrm{c}$ & $49.19 \mathrm{c}$ \\
1.33 & $12.50 \mathrm{~cd}$ & $25.00 \mathrm{bc}$ & $37.50 \mathrm{bc}$ & $50.09 \mathrm{c}$ & $60.65 \mathrm{c}$ & $72.71 \mathrm{~d}$ & $43.08 \mathrm{~d}$ \\
0.66 & $9.167 \mathrm{~d}$ & $21.67 \mathrm{c}$ & $32.50 \mathrm{c}$ & $44.07 \mathrm{c}$ & $56.85 \mathrm{c}$ & $67.40 \mathrm{e}$ & $38.61 \mathrm{e}$ \\
Control & $0.00 \mathrm{e}$ & $0.00 \mathrm{~d}$ & $0.00 \mathrm{~d}$ & $0.00 \mathrm{~d}$ & $0.00 \mathrm{~d}$ & $0.00 \mathrm{f}$ & $0.00 \mathrm{f}$ \\
\hline LSD & 5.027 & 5.641 & 7.799 & 6.346 & 5.315 & 4.895 & 3.248 \\
CV $(\%)$ & 47.92 & 27.04 & 25.53 & 16.28 & 11.32 & 8.83 & 9.59 \\
s_ & 1.768 & 1.984 & 2.743 & 2.232 & 1.869 & 1.721 & 1.142 \\
$\mathrm{x}$ & & & & & & & \\
\hline
\end{tabular}

HAT $=$ Hour after treatment. Within column values followed by different letter(s) are significantly different at $5 \%$ level of probability by DMRT. 
Table 3. Toxicity effect of different plant extracts of different doses against $6^{\text {th }}$ instar larvae of Tribolium castaneum at different HAT (interaction of plant, dose and time).

\begin{tabular}{|c|c|c|c|c|c|c|c|c|}
\hline \multirow{2}{*}{$\begin{array}{c}\text { Plant extracts } \\
\text { used }\end{array}$} & \multirow{2}{*}{$\begin{array}{c}\text { Doses } \\
(\mathrm{mg} / \\
\left.\mathrm{cm}^{2}\right) \\
\end{array}$} & \multicolumn{7}{|c|}{ Percentage of larval mortality at different time intervals } \\
\hline & & 12HAT & 24HAT & 36HAT & 48HAT & 60HAT & 72HAT & $\begin{array}{l}\text { Average } \\
\text { mortality }\end{array}$ \\
\hline \multirow{6}{*}{ Neem } & 10.61 & $13.3 \mathrm{cdef}$ & $\begin{array}{l}33.33 \\
\text { de }\end{array}$ & $46.67 \mathrm{de}$ & $53.71 \mathrm{ef}$ & $62.97 \mathrm{c}$ & $\begin{array}{l}95.83 \\
a b\end{array}$ & $50.97 \mathrm{ghi}$ \\
\hline & & $10.0 \mathrm{defg}$ & 30.00 & 36.67 efg & $49.63 \mathrm{fg}$ & 55.56 & 78.57 & $43.41 \mathrm{j}$ \\
\hline & 5.30 & $6.66 \mathrm{efg}$ & $\begin{array}{l}\text { de } \\
16.67 \\
\text { fgh }\end{array}$ & $\begin{array}{l}26.67 \\
\text { fgh }\end{array}$ & $50.00 \mathrm{fg}$ & $\begin{array}{l}\text { cd } \\
48.15 \\
\text { de }\end{array}$ & $\begin{array}{l}\mathrm{cd} \\
64.88 \text { ef }\end{array}$ & $35.51 \mathrm{k}$ \\
\hline & 1.33 & $6.66 \mathrm{efg}$ & $13.33 \mathrm{gh}$ & $13.33 \mathrm{hi}$ & $17.78 \mathrm{i}$ & $29.63 \mathrm{f}$ & $39.28 \mathrm{~g}$ & $20.00 \mathrm{~m}$ \\
\hline & 0.66 & $3.33 \mathrm{fg}$ & $10.00 \mathrm{hi}$ & $13.33 \mathrm{hi}$ & $17.78 \mathrm{i}$ & $18.52 \mathrm{~g}$ & $26.19 \mathrm{~h}$ & $14.86 \mathrm{~m}$ \\
\hline & 0.00 & $0.00 \mathrm{~g}$ & $0.00 \mathrm{i}$ & $0.00 \mathrm{i}$ & $0.00 \mathrm{j}$ & $0.00 \mathrm{~h}$ & $0.00 \mathrm{i}$ & $0.00 \mathrm{n}$ \\
\hline \multirow{6}{*}{ Thorn apple } & 10.61 & $20.00 \mathrm{bcd}$ & $\begin{array}{l}36.67 \\
\mathrm{~cd}\end{array}$ & $\begin{array}{l}50.00 \\
\text { cde }\end{array}$ & $60.37 \mathrm{def}$ & $66.67 \mathrm{c}$ & $88.4 \mathrm{abc}$ & $\begin{array}{l}53.69 \\
\text { fghi }\end{array}$ \\
\hline & 5.30 & $13.33 \mathrm{cdef}$ & $\begin{array}{l}26.67 \\
\text { def }\end{array}$ & $43.33 \mathrm{def}$ & $50.00 \mathrm{fg}$ & $66.67 \mathrm{c}$ & $\begin{array}{l}84.72 \\
\text { bc }\end{array}$ & $47.45 \mathrm{ij}$ \\
\hline & 2.65 & $10.00 \mathrm{defg}$ & $\begin{array}{l}16.67 \\
\text { fgh }\end{array}$ & $\begin{array}{l}26.67 \\
\text { fgh }\end{array}$ & $39.26 \mathrm{gh}$ & $\begin{array}{l}48.15 \\
\text { de }\end{array}$ & $\begin{array}{l}68.98 \\
\text { de }\end{array}$ & $34.95 \mathrm{k}$ \\
\hline & 1.33 & $6.667 \mathrm{efg}$ & $10.00 \mathrm{hi}$ & $26.67 \mathrm{fgh}$ & $39.26 \mathrm{gh}$ & 40.74 ef & 61.58 ef & $30.82 \mathrm{kl}$ \\
\hline & 0.66 & $6.667 \mathrm{efg}$ & $6.667 \mathrm{hi}$ & $23.33 \mathrm{gh}$ & $31.85 \mathrm{~h}$ & 40.74 ef & $57.87 \mathrm{f}$ & 27.861 \\
\hline & 0.00 & $0.00 \mathrm{~g}$ & $0.00 \mathrm{i}$ & $0.00 \mathrm{i}$ & $0.00 \mathrm{j}$ & $0.00 \mathrm{~h}$ & $0.00 \mathrm{i}$ & $0.00 \mathrm{n}$ \\
\hline \multirow{6}{*}{ Custard apple } & 10.61 & $20.00 \mathrm{bcd}$ & $\begin{array}{l}33.33 \\
\text { de }\end{array}$ & $\begin{array}{l}56.67 \\
\text { bcd }\end{array}$ & $70.00 \mathrm{bcd}$ & $96.67 \mathrm{a}$ & $100.0 \mathrm{a}$ & $62.78 \mathrm{cde}$ \\
\hline & 5.30 & 16.6 bcde & $\begin{array}{l}30.00 \\
\text { de }\end{array}$ & $\begin{array}{l}56.67 \\
\text { bcd }\end{array}$ & $66.67 \mathrm{cde}$ & $\begin{array}{l}92.59 \\
\mathrm{ab}\end{array}$ & $100.0 \mathrm{a}$ & $60.43 \mathrm{def}$ \\
\hline & 2.65 & 16.6 bcde & $\begin{array}{l}30.00 \\
\text { de }\end{array}$ & $\begin{array}{l}53.33 \\
\text { cde }\end{array}$ & $63.33 \mathrm{cdef}$ & $\begin{array}{l}85.93 \\
a b\end{array}$ & $\begin{array}{l}92.96 \\
a b\end{array}$ & $57.04 \mathrm{efg}$ \\
\hline & 1.33 & $13.33 \mathrm{cdef}$ & $\begin{array}{l}30.00 \\
\text { de }\end{array}$ & $\begin{array}{l}50.00 \\
\text { cde }\end{array}$ & 60.00 def & $82.59 \mathrm{~b}$ & $\begin{array}{l}93.33 \\
a b\end{array}$ & $54.88 \mathrm{fgh}$ \\
\hline & 0.66 & $10.00 \mathrm{defg}$ & $23.33 \mathrm{efg}$ & $36.67 \mathrm{efg}$ & $50.00 \mathrm{fg}$ & $81.85 \mathrm{~b}$ & $92.96 \mathrm{ab}$ & 49.13 hij \\
\hline & 0.00 & $0.00 \mathrm{~g}$ & $0.00 \mathrm{i}$ & $0.00 \mathrm{i}$ & $0.00 \mathrm{j}$ & $0.00 \mathrm{~h}$ & $0.00 \mathrm{i}$ & $0.00 \mathrm{n}$ \\
\hline \multirow{9}{*}{ Castor } & 10.61 & $33.33 \mathrm{a}$ & $60.00 \mathrm{a}$ & $76.67 \mathrm{a}$ & $90.00 \mathrm{a}$ & $96.67 \mathrm{a}$ & $96.67 \mathrm{a}$ & $75.56 \mathrm{a}$ \\
\hline & 5.30 & $33.33 \mathrm{a}$ & $\begin{array}{l}56.67 \\
a b\end{array}$ & $73.33 \mathrm{ab}$ & $86.67 \mathrm{a}$ & $\begin{array}{l}92.96 \\
a b\end{array}$ & $\begin{array}{l}92.96 \\
a b\end{array}$ & $72.65 \mathrm{ab}$ \\
\hline & 2.65 & $26.67 \mathrm{ab}$ & $\begin{array}{l}53.33 \\
\mathrm{ab}\end{array}$ & $\begin{array}{l}66.67 \\
\mathrm{abc}\end{array}$ & $83.33 \mathrm{ab}$ & $\begin{array}{l}89.26 \\
a b\end{array}$ & $96.30 \mathrm{a}$ & $69.26 \mathrm{abc}$ \\
\hline & 1.33 & $23.33 \mathrm{abc}$ & $\begin{array}{l}46.67 \\
b c\end{array}$ & $\begin{array}{l}60.0 \\
\text { abcd }\end{array}$ & $83.33 \mathrm{ab}$ & $\begin{array}{l}89.63 \\
a b\end{array}$ & $96.67 \mathrm{a}$ & $66.61 \mathrm{bcd}$ \\
\hline & 0.66 & 16.6 bcde & $\begin{array}{l}46.67 \\
b c\end{array}$ & $\begin{array}{l}56.67 \\
\text { bcd }\end{array}$ & $76.67 \mathrm{abc}$ & $\begin{array}{l}86.30 \\
a b\end{array}$ & $\begin{array}{l}92.59 \\
a b\end{array}$ & $62.59 \mathrm{cde}$ \\
\hline & 0.00 & $0.00 \mathrm{~g}$ & $0.00 \mathrm{i}$ & $0.00 \mathrm{i}$ & $0.00 \mathrm{j}$ & $0.00 \mathrm{~h}$ & $0.00 \mathrm{i}$ & $0.00 \mathrm{n}$ \\
\hline & LSD & 10.05 & 11.28 & 15.60 & 12.69 & 10.63 & 9.789 & 6.496 \\
\hline & $\begin{array}{l}\mathrm{CV} \\
(\%)\end{array}$ & 47.92 & 27.04 & 25.53 & 16.28 & 11.32 & 8.83 & 9.59 \\
\hline & $\mathrm{s}_{-}$ & 3.536 & 3.967 & 5.486 & 4.464 & 3.738 & 3.443 & 2.285 \\
\hline
\end{tabular}

HAT $=$ Hour after treatment. Within column values followed by different letter(s) are significantly different at $5 \%$ level of probability by DMRT. 
against male moth larvae, Plodia interpunctella HBN (Lepidoptera: Phycitinae). Castor bean oil and pure compounds of $R$. communis had been reported to exhibit high toxic effects in target animals (Kumar et al. 2007). Castor bean also contains the alkaloid ricinin, the polyphenolic molecule epicatechin and fatty acids in their leaves (Zahir et al. 2012) all which have insecticidal properties. The biological activity of castor plant extracts might be attributed to its alkaloid contents such as saponins, lectins, trypsin inhibitor etc. which caused mortality to the larvae.

Table 4. Relative toxicity (probit analysis) of different plant extracts treated against $6^{\text {th }}$ instar larvae of Tribolium castaneum after 12, 24, 36, 48, 60 and 72 HAT.

\begin{tabular}{|c|c|c|c|c|c|}
\hline \multirow{2}{*}{$\begin{array}{c}\text { Plant extracts } \\
\text { used }\end{array}$} & \multirow{2}{*}{$\begin{array}{c}\text { No of } \\
\text { larvae } \\
\text { used }\end{array}$} & \multirow{2}{*}{$\begin{array}{c}\mathrm{LD}_{50} \text { values } \\
\left(\mathrm{mg} / \mathrm{cm}^{2}\right)\end{array}$} & \multicolumn{2}{|c|}{ 95\% fiducially limits } & \multirow{2}{*}{$\chi^{2}$ values $(\mathrm{df})$} \\
\hline & & & Lower & Upper & \\
\hline \multicolumn{6}{|c|}{ 12HAT } \\
\hline Neem & 30 & 1321.64 & 0.29 & 5856718 & $0.27(3)$ \\
\hline Thorn apple & 30 & 2464.44 & 0.02 & $2.916262 \mathrm{E}^{+08}$ & $0.13(3)$ \\
\hline Custard apple & 30 & 443.13 & 1.16 & 168276.4 & $0.24(3)$ \\
\hline Castor & 30 & 77.75 & 1.27 & 4730.86 & $0.26(3)$ \\
\hline \multicolumn{6}{|c|}{ 24HAT } \\
\hline Neem & 30 & 37.25 & 5.02 & 276.12 & $0.41(3)$ \\
\hline Thorn apple & 30 & 4105.10 & $1.98 \mathrm{E}^{-06}$ & $8.4938 \mathrm{E}^{+12}$ & $0.28(3)$ \\
\hline Custard apple & 30 & 23.47 & 6.43 & 85.67 & $0.08(3)$ \\
\hline Castor & 30 & 1.50 & 0.26 & 8.59 & $0.14(3)$ \\
\hline \multicolumn{6}{|c|}{ 36HAT } \\
\hline Neem & 30 & 12.78 & 4.95 & 33.03 & $0.66(3)$ \\
\hline Thorn apple & 30 & 2.37 & 0.77 & 7.32 & $0.53(3)$ \\
\hline Custard apple & 30 & 12.91 & 3.07 & 54.17 & $0.87(3)$ \\
\hline Castor & 30 & 0.32 & 0.03 & 3.31 & $0.01(3)$ \\
\hline \multicolumn{6}{|c|}{ 48HAT } \\
\hline Neem & 30 & 5.82 & 3.15 & 10.77 & $3.61(3)$ \\
\hline Thorn apple & 30 & 0.47 & 0.04 & 5.04 & $0.16(3)$ \\
\hline Custard apple & 30 & 4.55 & 1.90 & 10.91 & $0.51(3)$ \\
\hline Castor & 30 & 0.0096 & $4.07 \mathrm{E}^{-06}$ & 22.99 & $0.08(3)$ \\
\hline \multicolumn{6}{|c|}{ 60HAT } \\
\hline Neem & 30 & 4.07 & 2.42 & 6.84 & $0.72(3)$ \\
\hline Thorn apple & 30 & 0.049 & 0.001 & 1.61 & $0.83(3)$ \\
\hline Custard apple & 30 & 2.02 & 0.97 & 4.19 & $1.30(3)$ \\
\hline Castor & 30 & 0.0093 & $1.08 \mathrm{E}^{-05}$ & 8.15 & $0.28(3)$ \\
\hline \multicolumn{6}{|c|}{$72 \mathrm{HAT}$} \\
\hline Neem & 30 & 1.67 & 1.24 & 2.26 & $0.84(3)$ \\
\hline Thorn apple & 30 & 0.002 & $5.79 \mathrm{E}^{-08}$ & 74.72 & $0.21(3)$ \\
\hline Custard apple & 30 & 0.96 & 0.55 & 1.67 & $1.07(3)$ \\
\hline Castor & 30 & $1.7 \mathrm{E}^{-07}$ & $5.88 \mathrm{E}^{-32}$ & $5.07 \mathrm{E}^{+17}$ & $0.17(3)$ \\
\hline
\end{tabular}

HAT $=$ Hour after treatment

Values were based on five concentrations, three replications of 10 insects each.

$\chi^{2}=$ Goodness of fit.

The tabulated value of $\chi^{2}$ is 5.99 (d. $\mathrm{f}=2$ at $5 \%$ level). 
Probit analysis for direct toxic effect of different plant extracts against $6^{\text {th }}$ instar larvae of red flour beetle: Probit analysis for direct toxic effect of red flour beetle at 12, 24, 36, 48, 60 and 72 HAT of different plant extracts against $6^{\text {th }}$ instar larvae of red flour beetle are presented in Table 4 . Among the treatments, $\mathrm{LD}_{50}$ values at $12 \mathrm{HAT}$ indicated that castor $\left(77.75 \mathrm{mg} / \mathrm{cm}^{2}\right)$ plant extract was the most toxic followed by custard apple $(443.13$ $\left.\mathrm{mg} / \mathrm{cm}^{2}\right)$ plant extract while thorn apple plant extract $\left(2464.44 \mathrm{mg} / \mathrm{cm}^{2}\right)$ was the least toxic. Castor plant extract also maintained its highest toxicity when the $\mathrm{LD}_{50}$ values were compared at 24, 36, 48, 60 and $72 \operatorname{HAT}\left(1.50,0.32,0.0096,0.0093\right.$ and $1.72 \mathrm{E}^{-07}$ $\mathrm{mg} / \mathrm{cm}^{2}$ ). The chi-square values were insignificant at $5 \%$ level of probability of different plant extracts at different HAT and mortality data did not show any heterogeneity.

From the results of the probit analysis on the $6^{\text {th }}$ instar larvae of the red flour beetle, it is apparent that all the tested plants would be more or less effective for controlling red flour beetle but castor will be the most effective extracts against the $6^{\text {th }}$ instar larval of the red flour beetle. This result is in agreement with results reported by Ramos-Lopez et al. (2012) who evaluated the effect of ingested ricin oil, ricinin and hexanic, acetatoethylic and methanolic extracts from 16 to $24,000 \mathrm{ppm}$ on first instar Spodoptera frugiperda larva. All treatments with ricinin $(560 \mathrm{ppm})$ and acetatoethylic extracts $(1600 \mathrm{ppm})$ from R. communis seeds had reduced weight of the pupae by $21.6 \%$ to $4.9 \%$ respectively.

It may be concluded that the botanicals used in the present study had direct toxic effect on the $6^{\text {th }}$ instar larval of $T$. castaneum. Among the tested plants, castor extracts showed the highest toxic effect. The larvicidal potential of indigenous plant extracts against $T$. castaneum has good prospects. Moreover, additional studies are needed to develop appropriate formulation and application method of $R$. communis based pesticides against stored product pest.

\section{Acknowledgements}

The authors express gratitude to the University Grant Commission, Bangladesh, for financial support through the Institute of Research and Training, Hajee Mohammad Danesh Science and Technology University, Dinajpur.

\section{References}

Abbott, W.S. 1987. A method of computing the effectiveness of an insecticide. J. American Mosquito Cont. Asso. 3(2): 302-303.

Basheer, A.G.M. 2014. Ricinus communis (castor) as larvicide on Anopheles arabiensis Patton. Inter. J. Advan. pharma. Biol. Chem. 3(2): 319-328.

Busvine, J.R. 1971. A critical review of the techniques for test in insecticides. Communication of Agricultutal Bureau, (CAB) London, U. K. pp. 263-288.

Collavino, M., A. Pelicano and R.A. Giménez. 2006. Actividad insecticida de Ricinus communis L. sobre Plodia interpunctella HBN. (Lepidoptera: Phycitinae). Revista Facultad de Ciencias Agropecuarias Universidad Nacional de Cuyo. 38: 13-18. 
Larvicidal efficacies of four indigenous

Dubey, N.K., B. Srivastava and A. Kumar. 2008. Current status of plant product as botanical pesticides in storage pest management. J. Biopest. 1(2): 182-186.

Goodland, R., C. Watson and G. Ledec. 1985. Biocides bring positioning and pollution to third world. The Bangladesh Observer, $16^{\text {th }}$ and $17^{\text {th }}$ January 1995. p.3.

Isman, M.B. 2000. Plant essential oils for pest and disease management. Crop Prot. 19: 603-608.

Isman, M.B. 2006. Botanical insecticides, de-terrents, and repellents in modern agriculture and an increasingly regulated world. Ann. Review Entomol. 51: 45-66.

Joel, O.O. 2015. Efficacy of selected plant extracts against Tribolium castaneum Herbst in stored groundnut (Arachis hypogaea L.). African J. Plant Sci. 9(2): 90-96.

Khalequzzaman, M. and S. Sultana. 2006. Insecticidal activity of Annona squamosa L seed extracts against the red flour beetle, Tribolium castaneum (Herbst). J. Biol. Sci. 14: 107112.

Khan, A.R. and B.J. Selman. 1981. Some techniques for minimizing the difficulties in egg counting in Tribolium castaneum (Herbst). The Entomol. Record J. Vari. 93: 36-37.

Kumar, O., R.P.V. Lakshmana, S. Pradhan, R. Jayaraj, A.S. Bhaskar, A.B. Nashikkar and R. Vijayaraghavan. 2007. Dose dependent effect of ricin on DNA damage and antioxidant enzymes in mice. Cell and Mol. Biol. 53: 92-102.

Mahdeem, H. 1998. "Annona reticulata". Neglected Crops. Department of Horticulture \& Landscape Architecture, Purdue University. Archived from the original on 11 May 2008. Retrieved 16 April 2008.

Mishra, B.B., S.P. Tripathi and C.P.M. Tripathi. 2012a. Response of Tribolium Castaneum (Coleoptera: Tenebrionidae) and Sitophilus oryzae (Coleoptera: Curculionidae) to potential insecticide derived from essential oil of Mentha arvensis leaves. Biol. Agri. Hort. 28: $34-40$.

Mishra, B.B., S.P. Tripathi and C.P.M. Tripathi. 2012b. Repellent effect of leaves essential oil from Eucalyptus globules (Mirtaceae) and Ocimum basilicum (Lamiaceae) against two major stored grain insect pests of coleopterans. Nat. Sci. 10(2): 50-54.

Mondal, K.A.M.S.H. 1984. A method of determining the larval instars of Tribolium castaneum Herbst (Coleoptera: Tenebrionidae). Lab. Prac. 33(10): 120-121.

Morgan, E.D. 2009. Azadirachtin, a scientific gold mine. Bioorg. Med. Chem. 17(12): 4096-4105.

Park, T. 1962. Beetles, competition and population. Science 138: 1369-1375.

Pimentel, D., D. Andow, R. Dyson-Hudson. D. Gallahan, S. Jacobson, M. Irish, S. Croop, A. Moss, I. Schreiner, M. Shepard, T. Thompson and B. Vinzant. 1980. Environmental and social cost of pesticides. A preliminary assessment. Oikos. 34: 125-140.

Rajashekar, Y., N. Bakthavatsalam and T. Shivanandappa. 2012. Botanicals as grain protectants. Psyche. 2012: 1-13.

Rajendran, S. and V. Sriranjini. 2008. Plant products as fumigants for stored product insect control. J. Stored Prod. Res. 44: 126-135.

Ramos-Lopez, M.A., M.M. Gonzalez-Chavez, N.C. Cardenas-Ortega, M.A. Zavala-Sanchez and G.S. Perez. 2012. Activity of the main fatty acid components of the hexane leaf extract of R. communis against Spodoptera. frugiperda. African J. Biotech. 11: 4274-4278.

Sharaby, A. 1988. Evaluation of some myrtaceae plant leaves as protectants against the infestation by Sitophilus oryzae L. and Sitophilus granarius L. Insect Sci. Appl. 9: 465-468.

Siddiqui, B.S., S.T. Ali, R.M. Tariq, T. Gulzar, M. Rasheed and R. Mehmood. 2009. GC-based analysis of insecticidal constituents of the flower of Azadirachta indica A. Juss. Nat. Prod. Res. 23(3): 271-283. 
Tripathi, A.K., S. Upadhyay, M. Bhuiyan and P.R. Bhattacharya. 2009. A Review on Prospects of Essential Oils as Biopesticides in Insect-Pest Management. J. Pharmacol. Phyto. 1: 5263.

Yasir, M., M. Sagheer, M. Hasan, S.K. Abbas, S. Ahmad and Z. Ali. 2012. Growth, development and reproductive inhibition in the red flour beetle, Tribolium castaneum (Herbst) (Coleoptera: Tenebrionidae) due to larval exposure to flufenoxur on-treated diet. Asian J. Pharma. Biol. Res. 2(1): 51-5.

Zahir, A. A., A.A. Rahuman, A. Bagavan, K. Geetha, C. Kamaraj and G. Elango. 2012. Evaluation of medicinal plant extracts and isolated compound epicatechin from $R$. communis against Poromphistomum cervi. Parasitol. Res. 111(4):1629-35. 